Prof. Dr. Franz Riklin

\title{
Das Unmittelbarkeitsprinzip im schweizerischen Strafverfahrensrecht
}

\section{Vorbemerkung}

Die Schweiz kennt erst seit dem 1.1.2011 eine nationale Strafprozessordnung ${ }^{1}$, denn zuvor war das Strafprozessrecht schwergewichtig kantonal geregelt. Seither gibt es nun auch eine höchstrichterliche (d.h. bundesgerichtliche) Rechtsprechung zu den neuen Vorschriften. Früher gab es dies im Strafprozessbereich nur punktuell, weil kantonale Vorschriften und deren Einhaltung vor dem Bundesgericht nur gerügt werden konnten, wenn Bundesrecht tangiert war, namentlich die Bundesverfassung sowie die Europäische Menschenrechtskonvention (EMRK).

\section{Das Unmittelbarkeitsprinzip im Kontext der verschiedenen Verfahrenserledigungsarten}

Das Unmittelbarkeitsprinzip spielt bei gerichtlichen Hauptverhandlungen eine Rolle. Bei einer rechtsvergleichenden Betrachtung sind deshalb jeweils auch andere Möglichkeiten der Verfahrenserledigung anklagetauglicher Verfahren $\mathrm{zu}$ beachten, bei denen das Unmittelbarkeitsprinzip keine Rolle spielt, als jene, die über gerichtliche Urteile erfolgen. Während in Deutschland sehr grob beurteilt gut 50\% solcher Verfahren durch Diversion erledigt werden, d.h. Verfahrenseinstellungen aus Opportunität mit oder ohne Auflagen, ferner etwa 25\% über Strafbefehle, die mangels Einspruch in Rechtskraft erwachsen und etwa 25\% durch gerichtliche Urteile, ist in der Schweiz diese Aufteilung wesentlich anders. Es hat eine insgesamt problematische „Ökonomisierung“ des Strafprozesses eingesetzt. Verfahrenseinstellungen aus Gründen der Opportunität sind wegen der Sorge um die

1 www.admin.ch/ch/d/as/2010/1881.pdf

Franz Riklin: emeritierter Professor für Straf- und Strafprozessrecht der Universität Fribourg 
gleichmäßige Durchsetzung des Strafrechts nur unter sehr engen Voraussetzungen möglich (Art. 8 sowie 319 Abs. 1 lit. e und Abs. 2 StPO) und haben keinen großen Entlastungseffekt. Das hat aber umgekehrt dazu geführt, dass das Schwergewicht der Erledigung anklagetauglicher Verfahren beim Strafbefehlsverfahren liegt. Solche Verfahren sind bei geringfügigeren Delikten und namentlich pekuniären Sanktionen zwar vertretbar, doch wird in der Schweiz diese Verfahrenserledigungsart viel großzügiger gehandhabt als in Deutschland und ist an lockerere Regeln gebunden. Ein Strafbefehl wird ohne Beteiligung eines Richters nur durch die Staatsanwaltschaft erlassen und es können Freiheitsstrafen bis zu sechs Monaten mit oder ohne Bewährung verhängt werden (Art. 352 StPO). Außer in Sonderfällen muss ein Strafbefehl nicht einmal begründet werden (folgt indirekt aus Art. 353 Abs. 1 lit.f StPO und Art. 41 Abs. 2 StGB). Laut Statistikamt waren 2010 fast 60\% aller Urteile Folge rechtskräftig gewordener Strafbefehle; mehrere Autoren schätzen den Anteil sogar auf bis zu 98\% ein². Dies ist nur eine scheinbare Differenz, weil sich das Statistikamt auf Verurteilungen wegen Verbrechen und Vergehen beschränkt und Übertretungen nicht einbezieht. Das Strafbefehlsverfahren (ohne Einspruch) ist somit zum Normalfall eines Strafprozesses geworden. Im Ergebnis hat sich in diesem Bereich ein „neoinquisitorisches“ Verfahren mit der Staatsanwaltschaft als Dominatorin breit gemacht. Diese Entwicklung findet auch im Jargon vieler Medien ihren Niederschlag. Man kann z.B. lesen, eine Staatsanwaltschaft habe jemanden zu 30 Tagessätzen Geldstrafe verurteilt, auch wenn es sich vorerst nur um den Strafbefehl eines Staatsanwalts handelt. Und die Politik fordert für Hooligans „Schnellgerichte“, die z.B. bei Ausschreitungen in Fußballstadien noch vor Ort Strafen aussprechen sollen, obwohl es sich höchstens um „Schnell-Staatsanwälte“ handeln kann, die bestenfalls unverzüglich Strafbefehle erlassen könnten, aber dies auch nur bei klarer Beweislage.

Zudem hat das mit der neuen StPO eingeführte sog. „Abgekürzte Verfahren“ (Art. 358ff. StPO), das ursprünglich vor allem für komplexe Fälle im Bereich der Wirtschaftskriminalität gedacht war, trotz überwiegend kritischer Aufnahme in der Doktrin eine ungeahnte Verbreitung erfahren. Danach können sich Beschuldigter und Staatsanwaltschaft unter Vermeidung eines in die Details gehenden Vorverfahrens über den Inhalt der Anklage und die Sanktion einigen. Bedingung ist, dass der Beschuldigte den wesentlichen Sachverhalt eingesteht, höchstens eine Freiheitsstrafe von fünf Jahren zur Diskussion steht und die Zivilansprüche zumindest im Grundsatz anerkannt sind. Die Absprache muss dem Gericht zur

2 Vgl. mit weiteren Verweisen Riklin, Der Strafbefehl im deutschen und im schweizerischen Strafprozessrecht - eine rechtsvergleichende Betrachtung, in: Müller-Dietz et al., Festschrift für Heike Jung, Baden-Baden 2007, S. 761 ff.; Daphinoff, Das Strafbefehlsverfahren in der Schweizerischen Strafprozessordnung, Zürich 2012, S. $59 \mathrm{f}$. 
Genehmigung überwiesen werden, das darüber nach einer grundsätzlich öffentlichen Hauptverhandlung zu befinden hat, an der eine summarische Überprüfung ohne Beweisverfahren erfolgt. Dabei ist auch $\mathrm{zu}$ prüfen, ob die beantragten Sanktionen angemessen sind (Art. 362 Abs. 1 lit. c StPO). Zahlenmäßig haben solche Fälle stark zugenommen, vor allem im Bereich der Drogenkriminalität ${ }^{3}$. Bei einem seltenen ablehnenden Entscheid, der nicht anfechtbar ist, geht der Fall an die Staatsanwaltschaft zur Durchführung eines ordentlichen Verfahrens zurück (Art. 362 Abs. 3 StPO).

\section{III. Öffentliche Hauptverhandlungen als Ausnahme und kompensatorische Vorkehren}

Das Schweizerische Bundesgericht hat zwar die Wichtigkeit des Grundsatzes der Öffentlichkeit betont: In BGE 124 IV $238^{4}$ z. B. wird gesagt, die Öffentlichkeit der Verhandlung sorge für Transparenz in der Rechtspflege, die eine demokratische Kontrolle durch das Volk erst ermögliche und als wesentliches Element des Rechts auf ein faires Verfahren zu den Grundlagen eines demokratischen Rechtsstaates gehöre. Dennoch ist, wie erwähnt, die überwiegende Mehrzahl von Verfahren, die zu Verurteilungen führen, nicht öffentlich oder - soweit das abgekürzte Verfahren zur Diskussion steht - für die Öffentlichkeit nur sehr beschränkt transparent.

Erwähnenswert ist immerhin, dass kompensatorisch eine gewisse „Entheimlichung“ des Vorverfahrens erfolgen kann. Gemäß Art. 69 Abs. 2 StPO können interessierte Personen in einen rechtskräftig gewordenen Strafbefehl Einsicht nehmen. Dies gilt auch für Medienvertreter, die ihn kritisch würdigen können. Das Bundesgericht hat darüber hinaus in BGE 134 I 286 in begründeten Fällen grundsätzlich ein solches Einsichtsrecht von Interessierten auch auf Einstellungsund sog. Nichtanhandnahmeverfügungen ausgedehnt, wenn ein schutzwürdiges

$3 \mathrm{Zu}$ ersten Erfahrungen vgl. Bürgisser, Erste Erfahrungen mit dem abgekürzten Verfahren (Art. 358-362 StPO) in der Praxis, Justice - Justiz - Giustizia (Richterzeitung) 2012/3, diesen Aufsatz findet man in google.ch unter „Martin Bürgisser, Richterzeitung“. Leider wird die Zahl dieser Verfahren gesamtschweizerisch noch nicht erfasst. In einzelnen Kantonen ist von einem Anteil von $10 \%$ und sogar von $25 \%$ der Hauptverhandlungen die Rede, die im abgekürzten Verfahren durchgeführt werden.

4 Bundesgerichtsentscheide (BGE) findet man unter http://www.bger.ch/index.htm in der Rubrik Rechtsprechung (gratis), BGE (Leitentscheide und Urteile EGMR) Index der Bundesgerichtsund EGMR-Entscheide. 
Interesse an der Klärung der Frage bestehe, weshalb es zur nichtgerichtlichen Verfahrenserledigung ohne Straffolgen durch Sach- und Prozessentscheide gekommen sei, wobei sich das schutzwürdige Informationsinteresse aus Gründen der Überwachung der Justiz - bei Medien aus deren Kontrollfunktion - ergeben könne (BGE 137 I 16).

\section{Geltung eines bloß beschränkten Unmittelbarkeitsprinzips}

Im geltenden Recht ist das Unmittelbarkeitsprinzip, soweit es nach dem Gesagten noch zum Zuge kommt, nur beschränkt verwirklicht. Das hat - wie unter Ziff. V. noch aufgezeigt wird - seine Gründe.

Die wichtigste Regel ist Art. 350 Abs. 2 StPO: danach berücksichtigt das Gericht bei der Urteilsberatung die im Vorverfahren und im Hauptverfahren erhobenen Beweise. Dies bedeutet, dass sich das Gericht auch auf das Ergebnis des Vorverfahrens und damit auf die Akten stützen kann. Der Transfer von Informationen aus dem Vorverfahren in die Hauptverhandlung und Vorhalte aus den Ermittlungsakten sind deshalb problemlos möglich. Auf Einschränkungen wird unter Ziff. V. zurückzukommen sein. Protokolle der Einvernahme von Zeugen z. B. dürfen als Beweismittel nicht nur dann verwendet werden, wenn eine unmittelbare Befragung in der Hauptverhandlung nicht möglich ist. Bei Kollegialgerichten setzt die Verfahrensleitung im Rahmen der Vorbereitung der Hauptverhandlung zudem die Akten in Zirkulation (Art. 330 Abs. 2 StPO), weshalb alle Richter schon vor Beginn der Hauptverhandlung volle Aktenkenntnis haben. Die unmittelbare Beweisaufnahme in der Hauptverhandlung gilt nur als einer der möglichen Wege zur Wahrheitserforschung neben der direkten Verwertung der Untersuchungsakten.

Was das Beweisverfahren in der Hauptverhandlung anbetrifft, besagt Art. 343 StPO:

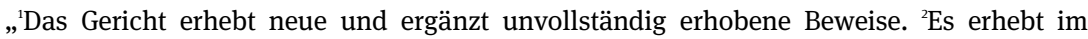
Vorverfahren nicht ordnungsgemäss erhobene Beweise nochmals. ${ }^{3}$ Es erhebt im Vorverfahren ordnungsgemäss erhobene Beweise nochmals, sofern die unmittelbare Kenntnis des Beweismittels für die Urteilsfällung notwendig erscheint“.

Dies bedeutet:

- Neue Beweise werden an der Hauptverhandlung (nur) erhoben, wenn das bisher nicht geschehen ist und eine Beweiserhebung nach Auffassung des Gerichts nötig ist. 
- Eine unvollständig erhobene Beweiserhebung liegt z. B. vor, wenn ein Zeuge in der Untersuchung zwar befragt wurde, aber man ihm wichtige Fragen nicht stellte oder wenn keine kontradiktorische Befragung stattfand. Laut Bundesgericht ist dem Konfrontationsrecht gemäß Art. 6 Abs. 3 lit. d EMRK Rechnung getragen, wenn der Beschuldigte mindestens einmal im Verfahren also entweder in der Untersuchung oder in der erstinstanzlichen gerichtlichen Hauptverhandlung - Gelegenheit gehabt hat, an den Beweiserhebungen mitzuwirken ${ }^{5}$. Zur Parteiöffentlichkeit im Vorverfahren nachstehend unter $\mathrm{V}$.

- Eine im Vorverfahren an sich korrekt erhobene Zeugenaussage ist nur dann $\mathrm{zu}$ wiederholen, wenn das Protokoll dem Gericht nicht ausreichend und zuverlässig erscheint, namentlich wenn es auf den unmittelbaren persönlichen Eindruck ankommt.

Unmittelbar ist das Verfahren in Bezug auf die Einvernahme des Beschuldigten, der in aller Regel (d.h. bei Verbrechen und Vergehen) verpflichtet ist, an der Hauptverhandlung teilzunehmen (Art. 336 Abs. 1 StPO) und, wie dargelegt, in Bezug auf umstrittene Beweise, die an der Hauptverhandlung abgenommen bzw. wiederholt werden.

\section{Das beschränkte Unmittelbarkeitsprinzip im Kontext des gesamten prozessualen Instrumentariums}

Die Regelung über die (beschränkte) Unmittelbarkeit darf nicht gänzlich isoliert, sondern muss im Kontext des gesamten Instrumentariums gesehen werden, das eine Strafprozessordnung im Interesse eines fairen, der Wahrheitsfindung dienenden und in angemessener Zeit durchzuführenden Verfahrens zur Verfügung stellt. Auch in der Schweiz geht man davon aus, dass unter Vorbehalt rechtsstaatlicher Schranken in Strafverfahren die materielle Wahrheit anzustreben ist. Entsprechend gilt der Untersuchungsgrundsatz (Art. 6 StPO), wonach die Strafbehörden von Amtes wegen alle für die Beurteilung der Tat und der beschuldigten Person bedeutsamen Tatsachen abzuklären und belastende wie entlastende Umstände mit gleicher Sorgfalt zu untersuchen haben. Als Strafbehörden gelten Strafverfolgungsbehörden (Polizei und Staatsanwaltschaft) sowie die aburteilen-

5 BGE 105 Ia 397; 118 Ia 469f.; 125 I 132f. 
den Behörden (Art. 12ff. StPO). Auch findet in der gerichtlichen Hauptverhandlung bei der Beweiserhebung kein adversarisches Verfahren à l'américaine statt, sondern die Durchführung der Einvernahmen obliegt gemäß Art. 341 StPO der Verfahrensleitung oder einem von ihr bestimmten Mitglied des Gerichts.

Was das Vorverfahren anbetrifft besteht in der Schweiz im Vergleich zu Deutschland eine ganz unterschiedliche Akzentsetzung. Übersprungen sei die Schilderung von Regelungen, wie sie auch anderswo in ähnlicher Art bestehen, wie etwa über die notwendige und die unentgeltliche Verteidigung. Verwiesen sei hingegen auf die folgenden Institute und Regelungen, die im Zusammenhang mit der Unmittelbarkeitsdiskussion besondere Beachtung verdienen und eine Beschränkung des Unmittelbarkeitsprinzips aus Schweizer Sicht zu rechtfertigen vermögen. Es geht um neun Aspekte:

1. Angesichts der vielfach für den Prozessausgang entscheidenden Bedeutung des Vorverfahrens existiert eine gut ausgebaute Regelung über die sog. Parteiöffentlichkeit. Sie gestattet es der Verteidigung, bereits an der ersten polizeilichen Einvernahme anwesend zu sein und Fragen zu stellen (sog. „Anwalt der ersten Stunde“, Art. 159 StPO). Anwaltsverbände haben deshalb für Dringlichkeitsfälle Pikettdienste eingerichtet. Auch kann die Verteidigung im Fall einer Festnahme oder der Untersuchungshaft grundsätzlich unbehindert mit dem Beschuldigten verkehren, außer bei begründetem Verdacht auf Missbrauch, wobei Einschränkungen der Genehmigung durch ein Gericht (das sog. Zwangsmaßnahmengericht ${ }^{6}$ ) bedürfen (Art. $235 \mathrm{Abs} .4 \mathrm{StPO}$ ). Die Rechtsmissbrauchsklausel stellt hohe Anforderungen an die Begründung einer Einschränkung. In der anschließenden eigentlichen Untersuchung ist es den Parteien (dem Beschuldigten stets und dem Geschädigten dann, wenn er ausdrücklich erklärt, sich am Strafverfahren als Straf- und/oder Zivilkläger beteiligen zu wollen, Art. 118f. StPO) sowie den jeweiligen Rechtsbeiständen erlaubt, sich an Verfahrenshandlungen (namentlich an Beweiserhebungen) zu beteiligen (Art. 107 Abs. 2, 147, 312 Abs. 2 StPO), wobei die Einschränkungsmöglichkeiten sehr restriktiv umschrieben werden (Art. 108 StPO). Zwar besteht im Vorverfahren kein Anspruch auf Verschiebung einer Beweiserhebung; die Partei oder ihr Rechtsbeistand können jedoch grundsätzlich (mit gewissen Ausnahmen) die Wiederholung der Beweiserhebung verlangen, wenn sie aus zwingenden Gründen an der Teilnahme verhindert waren. Beweise, die in Verletzung dieser Vorschrift erhoben worden sind, dürfen nicht zu Lasten der Partei verwertet werden, die nicht anwesend war (Art.147 StPO). Auch das Akteneinsichtsrecht ist liberal geregelt (zu den Schranken vgl. Art. 108 StPO).

6 Vgl. zu diesem nachstehend Ziffer 5. 
2. Als Beschuldigter gilt schon, wer in einer Strafanzeige, einem Strafantrag oder von einer Strafbehörde, wozu wie erwähnt auch die Polizei zählt, in einer Verfahrenshandlung einer Straftat verdächtigt wird (Art. 111 Abs. 1 StPO), wobei ein Anfangsverdacht ausreicht. Dann muss er nach Art. 158 StPO unverzüglich schon auf Polizeiebene - zu Beginn der ersten Einvernahme in einer ihm verständlichen Sprache über seine Rechte, namentlich sein Schweigerecht und sein Recht, einen Verteidiger beizuziehen sowie einen Übersetzer zu verlangen, unterrichtet werden. Und er muss auch darüber informiert werden, dass gegen ihn ein Vorverfahren eingeleitet worden ist und welche Straftaten Gegenstand dieses Verfahrens bilden. Ohne diese Hinweise ist eine Aussage nicht verwertbar. In der Schweiz ist man der Auffassung, dass ein Beschuldigter nach einer solchen qualifizierten Orientierung weiß, dass er Beschuldigter in einem Strafverfahren ist und es deshalb nicht einzusehen ist, warum z. B. eine Aussage im Vorverfahren, mit der er sich selbst belastet, in der Hauptverhandlung nicht verwertbar sein sollte.

3. Die Schweiz kennt ferner die ingeniöse Rechtsfigur der „Auskunftsperson“ (Art. 178 StPO). Diese Rolle kommt u.a. jemandem zu, der, ohne selber beschuldigt zu sein, als Täter oder Teilnehmer der abzuklärenden Straftat oder einer anderen damit zusammenhängenden Straftat nicht ausgeschlossen werden kann. Eine Auskunftsperson ist nicht zur Aussage verpflichtet, kann nicht wegen falschem Zeugnis bestraft werden und es gelten für sie sinngemäß die Bestimmungen über die Einvernahme des Beschuldigten (Art. 180 StPO).

4. Die personell vermutlich stärker als in Deutschland ausgestattete Staatsanwaltschaft muss die notwendigen Beweiserhebungen in der Untersuchung in der Regel selber durchführen, wobei sie nach deren Eröffnung die Polizei mit ergänzenden Ermittlungen beauftragen kann, die sich jedoch auf konkret umschriebene Abklärungen beschränken müssen (Art. 311f. StPO). Zwar wird im vorgängigen polizeilichen Ermittlungsverfahren eine selbständige Ermittlungstätigkeit der Polizei anerkannt, doch kann die Staatsanwaltschaft das Verfahren jederzeit an sich ziehen (Art. 307 Abs. 2 StPO). Zudem ist diese über schwere Straftaten sowie über andere schwerwiegende Ereignisse unverzüglich zu informieren (Art. 307 Abs. 1 StPO), was in der Regel zur Übernahme des Falls durch die Staatsanwaltschaft führt. Die Polizei untersteht ihrer Aufsicht und ihren Weisungen (Art. 15 Abs. 2 StPO). Und was wichtig ist: Die Staatsanwaltschaft kann Zeugen befragen, die sich diesfalls bei Falschaussagen wegen falschem Zeugnis strafbar machen. Und das Recht, jemanden als Zeugen zu befragen, kann auch qualifizierten Polizeiangehörigen verliehen werden (Art.142 Abs. 2 StPO).

5. Bestimmte Zwangsmaßnahmen können nur durch ein besonderes Gericht, das sog. Zwangsmaßnahmengericht (Art. 18 StPO), angeordnet werden oder bedürfen dessen Genehmigung; dies betrifft nicht nur die Untersuchungs- und 
Sicherheitshaft, sondern auch geheime Überwachungsmaßnahmen, d.h. die Überwachung des Post- und Fernmeldeverkehrs, die Überwachung mit technischen Überwachungsgeräten, die Überwachung von Bankbeziehungen und die verdeckte Ermittlung (Art. 272, 274, 281 Abs. 1 und 289 StPO).

6. Ferner kann gegen Verfügungen und Verfahrenshandlungen von Polizei und Staatsanwaltschaft (namentlich gegen Zwangsmaßnahmen und Einschränkungen des rechtlichen Gehörs) in einem schriftlichen Verfahren Beschwerde an eine richterliche Beschwerdeinstanz erhoben werden. Rügbar sind Rechtsverletzungen (einschließlich der Überschreitung und des Missbrauchs des Ermessens sowie Rechtsverzögerung), die unvollständige oder unrichtige Feststellung des Sachverhalts und Unangemessenheit (Art. 394 StPO). Dies gilt allerdings nicht für die Anklageerhebung sowie aus Gründen der Prozessökonomie und der Verfahrensbeschleunigung für die Ablehnung von Beweisanträgen durch die Staatsanwaltschaft, wenn der Antrag ohne Rechtsnachteil vor dem erstinstanzlichen Gericht wiederholt werden kann (Art. 394 StPO).

7. In Bezug auf die Möglichkeit der Ablehnung von Beweisanträgen besteht eine weniger rigide Regelung als in Deutschland. Verwiesen sei auf die allgemeine (negative) Beweiserhebungsregel des Art. 139 StPO, wonach über Tatsachen, die unerheblich, offenkundig, der Strafbehörde bekannt oder bereits rechtsgenügend erwiesen sind, nicht Beweis geführt wird. Abgelehnte Beweisanträge können im Hauptverfahren erneut gestellt werden; sie sind somit im Fall der Ablehnung, wie bereits erwähnt, nicht unmittelbar anfechtbar (Art. 139 Abs. 3 StPO). Ganz allgemein ist der Gehörsanspruch gemäß bundesgerichtlicher Praxis verletzt, wenn ohne sachliche Gründe einem Beweismittel von vornherein jede Erheblichkeit abgesprochen wird (BGE 106 II 171). Andererseits wird auch vom Bundesgericht eine antizipierte Beweiswürdigung geschützt ${ }^{7}$.

8. Was die Übernahme der neueren Straßburger Rechtsprechung zum Konfrontationsrecht gemäß Art. 6 Abs. 3 lit. d EMRK anbetrifft, wonach die beschuldigte Person das Recht hat, Fragen an Belastungszeugen zu stellen oder stellen zu lassen, gilt folgendes:

Laut Bundesgericht ist der EMRK - wie bereits erwähnt - Rechnung getragen, wenn der Beschuldigte mindestens einmal im Verfahren, also entweder in der Untersuchung oder in der erstinstanzlichen gerichtlichen Verhandlung, Gelegenheit gehabt hat, an den Beweiserhebungen mitzuwirken. Ist dies im Vorverfahren geschehen, besteht vielfach kein Grund zur Wiederholung der Einvernahme in der Hauptverhandlung. Das Problem bilden jedoch gesetzlich geregelte Einschränkungen bei sog. Schutzmaßnahmen zugunsten besonders sensibler und be-

7 Vgl. z.B. BGE 115 Ia 101. 
sonders gefährdeter Zeugen sowie verdeckten Ermittlern (Art. 149-156 StPO). Dort gibt es Konstellationen, bei denen das Konfrontationsrecht eingeschränkt oder gar aufgehoben wird.

Problematisieren kann man in diesem Zusammenhang bereits die Gleichwertigkeit einer konventionellen mit einer simultanen Befragung, die mittels Videoübertragung aus einem separaten Raum (in dem sich der Beschuldigte befindet) in den Gerichtssaal erfolgt (weil man im Rahmen von Schutzmaßnahmen die Begegnung mit der zu befragenden Person vermeiden will), oder wenn die Befragung aus Distanzgründen mittels Videokonferenz stattfindet. Das geltende Recht enthält keine Restriktion. Nach Art. 76 Abs. 4 StPO kann die Verfahrensleitung, d.h. bis zur Anklageerhebung die Staatsanwaltschaft, Verfahrenshandlungen zusätzlich zur schriftlichen Protokollierung in Ton oder Bild festhalten und muss dies den anwesenden Personen lediglich vorgängig bekannt geben. Ihre Zustimmung ist nicht erforderlich. Dies gilt auch bei Einvernahmen mittels Videokonferenz (Art. 78 Abs. 6 StPO). Solche „Audiovisualisierungen“ halten sich jedoch in der Praxis bisher in Grenzen.

Einschneidender kann eine Einschränkung sein, wenn die beschuldigte Person von der Verhandlung ausgeschlossen wird und Fragen nur durch die Verteidigung stellen lassen kann. Eine andere Einschränkung besteht im Fall der Anonymisierung der befragten Person, indem man ihre Personalien verheimlicht oder sie zusätzlich durch Vermummung und/oder Stimmveränderung cachiert. Man kann sich fragen, ob bei einer völligen Anonymisierung und einer derartigen Aushöhlung des Konfrontationsrechts noch von einem fairen Verfahren gesprochen werden kann. Ebenfalls sehr stark eingeschränkt ist das Frage- und Konfrontationsrecht, wenn - wie etwa in Rechtshilfeverfahren - nur eine nichtsimultane Befragung auf dem Korrespondenzweg in Abwesenheit der Parteien durch einen vorgängig erstellten Fragenkatalog und/oder nachträglich gestellte Fragen gestützt auf die Lektüre des Protokolls ermöglicht wird. In der Lehre wird z.T. kritisch eingewendet, das Recht, direkt Fragen zu stellen und dadurch auf den Inhalt einer Aussage aktiv einzuwirken und insbesondere auch Mängel der Aussagequalität aufzudecken, gehöre zum Kerngehalt des Konfrontationsrechts und die Simultanität der Befragung sei eine notwendige Voraussetzung ${ }^{8}$. Allerdings ist gemäß dem Wortlaut von Art. 6 Abs. 3 lit. d EMRK auch vom Recht die Rede, (nur) Fragen an Belastungszeugen stellen zu lassen.

8 Vgl. Schleiminger, Konfrontation im Strafprozess, Art. 6 Ziff. 3 lit. d EMRK mit besonderer Berücksichtigung des Verhältnisses zum Opferschutz im Bereich von Sexualdelikten gegen Minderjährige, Basel 2001, S. 315 ff.; dies., Das Konfrontationsrecht des Angeklagten nach Art. 6 Ziff. 3 lit. d EMRK, Bemerkungen zu BGE 125 I 127, AJP (Aktuelle juristische Praxis) 1999, 1223 ff., 1231. 
Das Schweizerische Bundesgericht hat zu einigen dieser Konstellationen noch nie Stellung genommen, zu anderen schon. Ohne an dieser Stelle allzu sehr in die Details zu gehen, lässt sich sagen, dass es jeweils die maßgebenden Entscheide des EGMR zitiert und analysiert sowie im Einzelfall berücksichtigt hat. U.a. hat es (in einem Entscheid vor Inkrafttreten der StPO) ausgeführt, das Konfrontationsrecht sei dann absoluter Natur, wenn dem Beweismittel ausschlaggebende Bedeutung zukomme, d.h. wenn dieses den einzigen oder einen wesentlichen Beweis darstelle $^{9}$. In einem solchen Fall dürfe die Beantwortung von Fragen der Verteidigung an den Belastungszeugen auch nicht mittels antizipierter Beweiswürdigung für nicht notwendig erklärt werden. In der StPO selber findet man zu dieser Thematik der Schutzmaßnahmen acht Bestimmungen mit Floskeln wie:

- Die Verfahrensleitung könne die Verfahrensrechte der Parteien angemessen beschränken, wobei sie aber bei allen Schutzmaßnahmen für die Wahrung des rechtlichen Gehörs sorge, insbesondere die Verteidigungsrechte des Beschuldigten (Art. 149 Abs. 2 und 5 StPO).

- Bei sog. Opfern (Personen, die in ihrer physischen, psychischen oder sexuellen Integrität durch eine Straftat beeinträchtigt wurden) ist zwar auf Verlangen die Begegnung mit dem Beschuldigten zu vermeiden, wobei in diesem Fall dem Anspruch des Beschuldigten auf rechtliches Gehör auf andere Weise Rechnung zu tragen sei und eine Gegenüberstellung dennoch angeordnet werden könne, wenn der Anspruch auf rechtliches Gehör des Beschuldigten nicht auf andere Weise gewährleistet werden könne oder wenn ein überwiegendes Interesse der Strafverfolgung dies zwingend erfordere (Art. 152 Abs. 3 und 4 StPO).

- Bei Opfern von Straftaten gegen die sexuelle Integrität darf eine Gegenüberstellung mit dem Beschuldigten gegen den Willen des Opfers nur angeordnet werden, wenn der Anspruch des Beschuldigten auf rechtliches Gehör nicht auf andere Weise gewährleistet werden könne (Art. 153 Abs. 2 StPO).

Aus gesetzgeberischer Sicht ist es äußerst schwierig, die Grundsätze der Rechtsprechung des EGMR zum Konfrontationsrecht in die generell-abstrakte Form eines Gesetzes zu kleiden. Damit tut man sich auch in anderen Ländern schwer. Man müsste wohl eine Art Mustergesetz konzipieren. Dabei macht es einen Unterschied, ob es um die Argumentationsweise in einem höchstrichterlichen Entscheid geht, wo man im Einzelfall bei einer schwierigen Entscheidfindung z. B. auch noch prüfen kann, ob die Fairness des Verfahrens insgesamt gegeben war, während es aus gesetzgeberischer Sicht vergleichsweise problematischer wäre,

9 BGE 129 I $151 \mathrm{ff}$. 
eine entsprechende „overall fairness“ zu kodifizieren. Ohne sehr offene Ermessensklauseln wird man deshalb in diesem speziellen Bereich nicht auskommen.

9. Schließlich besteht ein vereinfachtes Rechtsmittelsystem, wonach alle Strafurteile erstinstanzlicher Gerichte mit dem Rechtsmittel der Berufung an die zweite (kantonale) Gerichtsinstanz weitergezogen werden können, die das Urteil in allen angefochtenen Punkten umfassend (d.h. in tatsächlicher und rechtlicher Hinsicht) überprüfen kann (Art. 398 Abs. 2 und 3 StPO), wobei auch neue Behauptungen und Beweise vorgebracht werden können. Es bestehen somit zwei Instanzen mit voller Kognition. Allerdings beruht das Rechtsmittelverfahren gemäß Art. 389 StPO auf den Beweisen, die im Vorverfahren und im erstinstanzlichen Hauptverfahren erhoben worden sind. Beweisabnahmen des erstinstanzlichen Gerichts werden nur wiederholt, wenn Beweisvorschriften verletzt worden sind, die Beweiserhebungen unvollständig waren oder die Akten über die Beweiserhebungen unzuverlässig erscheinen. Die Rechtsmittelinstanz erhebt dabei von Amts wegen oder auf Antrag einer Partei die erforderlichen zusätzlichen Beweise.

Eine Einschränkung gilt für den Fall, dass ausschließlich Übertretungen Gegenstand des erstinstanzlichen Hauptverfahrens bildeten; dann ist das Urteil nur wegen Rechtsfehlerhaftigkeit anfechtbar sowie wegen offensichtlich unrichtiger oder auf einer Rechtsverletzung beruhender Feststellung des Sachverhalts (Art. 398 Abs. 4 StPO).

Schließlich besteht eine dritte Instanz; gegen Urteile der Berufungsinstanz ist ein Weiterzug an das Schweizerische Bundesgericht möglich, das namentlich die Verletzung von Bundesrecht und von Völkerrecht überprüfen kann, Sachverhaltsfeststellungen hingegen nur, wenn sie offensichtlich unrichtig sind oder auf einer Rechtsverletzung beruhen.

\section{Schlussbemerkungen}

Man glaubt aus schweizerischer Sicht, eine beschränkte Unmittelbarkeit lasse sich angesichts der gut ausgebauten Parteiöffentlichkeit im Vorverfahren sowie anderen Regelungen und Institutionen, wie sie geschildert wurden, rechtfertigen. Es ist fraglich, ob die erheblichen Mehraufwendungen, die eine vollständige Umsetzung des Unmittelbarkeitsprinzips bedingen würde, mit einem entsprechenden Gewinn an Rechtsstaatlichkeit verbunden wären.

Pro memoria erwähnt sei, dass weder die schweizerische Bundesverfassung, noch die EMRK und auch nicht der Internationale Pakt über bürgerliche und politische Rechte (IPBPR) ein unmittelbares Verfahren vorschreiben. Hauptverhandlungen sind demzufolge in der Schweiz wesentlich kürzer als in Deutschland; wochenlange Hauptverhandlungen kommen nicht vor. 
Das Bedürfnis nach (informellen) Absprachen wird zudem stark zurückgedrängt. Zwar sind Deals bzw. Verständigungen auch in der Schweiz ein Thema, namentlich beim neu geschaffenen Institut des einleitend erwähnten abgekürzten Verfahrens sowie beim Strafbefreiungsgrund der Wiedergutmachung, wonach im Vorverfahren eine Einstellung möglich ist, wenn der Beschuldigte den Schaden gedeckt oder alle zumutbaren Anstrengungen unternommen hat, um das von ihm bewirkte Unrecht auszugleichen und wenn ferner die Voraussetzungen für die Aussetzung zur Bewährung erfüllt und das Interesse der Öffentlichkeit und des Geschädigten an der Strafverfolgung gering sind (Art. $53 \mathrm{StGB}$ ).

Auch wird mit diesem System die Problematik der unter Umständen fragwürdigen Qualität von Aussagen über weit zurückliegende Ereignisse in dem Ambiente einer öffentlichen Gerichtsverhandlung gemildert.

Dennoch ist der „schweizerische Weg“ auch innerhalb der Schweiz nicht unbestritten. Gegenstand von Kritik ist die Praxis, dass vielfach vom Recht, an der Hauptverhandlung Beweise abzunehmen, nur sehr zurückhaltend Gebrauch gemacht wird. Eine unmittelbare Beweisaufnahme findet abgesehen von der Befragung der beschuldigten Person meist nur in Ausnahmefällen statt. Umgekehrt halten sich mehrfache Vernehmungen derselben Zeugen in Grenzen. Die Hauptverhandlung dient weniger der Beweisaufnahme als vielmehr der Kontrolle und Sanktionierung des bereits im Vorverfahren Festgestellten. Gravierende Mängel des Vorverfahrens können zwar noch korrigiert werden, die maßgebenden Tendenzen sind aber bereits vorgegeben ${ }^{10}$. Doch in der Tat ist es so: Wird das Vorverfahren optimiert, ändert dies die Funktion der Hauptverhandlung und lädt zu Einschränkungen des Unmittelbarkeitsgebots ein.

\footnotetext{
Anmerkung: Schriftliche und leicht überarbeitete Fassung eines Referats, gehalten auf der internationalen Tagung des Arbeitskreises deutscher, österreichischer und schweizerischer Strafrechtslehrer (Arbeitskreis AE) am 29. September 2012 in Hannover. Sehr lesenswert ist auch die nach diesem Referat 2013 publizierte Luzerner Dissertation von Kaufmann, Das Unmittelbarkeitsprinzip und die Folgen seiner Einschränkung in der Schweizer Strafprozessordnung, Luzerner Beiträge zur Rechtswissenschaft, Band 71, Zürich/Basel/Genf 2013.
}

10 So Oberholzer, Grundzüge des Strafprozessrechts, dargestellt am Beispiel des Kantons St. Gallen, 2. Aufl. Bern 2005, S. 324f., und sinngemäß bestätigt in der 3. Auflage, Bern 2012, N. 1459. 